Journal of Animal and Veterinary Advances 11 (8): 1080-1086, 2012

ISSN: $1680-5593$

(C) Medwell Journals, 2012

\title{
Molecular Cloning, mRNA Expression and Characterization of a Novel FAIMI Gene from Chinese Banna Mini-Pig Inbred Line (BMI)
}

\author{
${ }^{1,2,3}$ Jinlong Huo, ${ }^{1,2}$ Pei Wang, ${ }^{4}$ Yue Zhao, ${ }^{3,}{ }^{4}$ Hailong Huo, ${ }^{4}$ Lixian Liu, \\ ${ }^{4}$ Rui Wang, ${ }^{3}$ Heng Xiao and ${ }^{1,2}$ Yangzhi Zeng \\ ${ }^{1}$ Key Laboratory of Banna Mini-Pig Inbred Line of Yunnan Province, \\ 650201 Kunming, Yunnan, China \\ ${ }^{2}$ Faculty of Animal Science and Technology, Yunnan Agricultural University, \\ 650201 Kunming, Yunnan, China \\ ${ }^{3}$ Faculty of Life Science, Yunnan University, 650091 Kunming, Yunnan, China \\ ${ }^{4}$ Department of Husbandry and Veterinary, \\ Yunnan Vocational and Technical College of Agriculture, 650212 Kunming, China
}

\begin{abstract}
The complete CDS sequence of Banna Mini-pig Inbred line (BMI) gene FAIM1 was amplified using the Reverse Transcriptase Polymerase Chain Reaction (RT-PCR) based on the conserved sequence information of the cattle or other mammals and known highly homologous swine ESTs. This novel gene was then deposited into NCBI database and assigned to Accession No.: JF271685. Sequence analysis revealed that the BMI FAIM1 encodes a protein of 200 amino acids that has high homology with the Fas apoptosis inhibitory molecule 1 proteins of five other species-cattle $95 \%$, horse $95 \%$, human $94 \%$, mouse $92 \%$ and rat $91 \%$. The phylogenetic tree analysis revealed BMI FAIM1 has a closer genetic relationship with the bovine FAIM1 than with those of horse, human, mouse and rat. Analysis by RT-PCR showed that BMI FAIM1 gene was over-expressed in lymph node, diencephalon, heart, muscle, moderately expressed in midbrain, spleen, lung, small intestine, fat and almost not expressed in other 9 tissues. Several microRNA target sites were predicted in the CDS of BMI FAIM1 mRNA for further studying this gene in the future.
\end{abstract}

Key words: Banna Mini-pig Inbred line (BMI), pig, FAIM1, tissue expression, bioinformatics analysis, China

\section{INTRODUCTION}

Fas Apoptosis Inhibitory Molecule (FAIM) is an highly evolutionarily conserved gene (from worm to fly to mouse to human) which had been reported to play a crital role in inhibiting death receptor-induced apoptosis in lymphocytes, neurons, hepatocytes and thymocytes in many species (Schneider et al., 1999; Segura et al., 2007; Choi et al., 2007; Huo et al., 2009, 2010). Besides apoptosis, it was also shown to be involved in NF-kB and Ras-ERK activation during neurite outgrowth (Sole et al., 2004). In addition, structural abnormalities of the human chromosomal location of FAIM are associated with B cell lymphoma, head and neck cancer, non small cell carcinoma of the lung and acute non-lymphocytic leukemia (Juneja et al., 1990; Cigudosa et al., 1999; Carey et al., 1993; Whang-Peng et al., 1991; Petersen et al., 1997; Pekarsky et al., 1995; Hamaguchi et al., 1997).

Swine are generally considered to be the most ideal biomedical laboratory animals for their anatomical, physiological and metabolic characteristics are similar to human's. Since 1950 s, some breeds of miniature swine have been developed in several countries such as Yucatan, Hanford, Sinclair, Pitman-Moore, Essex, Minnesota Hormel and Nebraska in the United States, Gottingen in Germany, Oh mini, Clawn and Huei-Jin in Japan and Corsica in France. They have been used as a major mammalian model for human studies because of the similarity in size and physiology and organ development and disease progression (Lunney, 2007; Brown and Terris, 1996; Douglas, 1972; Reeds and Odle, 1996; Swindle and Smith, 1998; Larsen and Rolin, 2004). The inbred animals are good enough to be used as experimental animals, owing to their clear genetic background, high homozygosity, stable inheritance and so on. Inbred animals can also make less experimental errors using in biological research than noninbred ones (Wright, 1921; Harris, 1997).

Based on above described about the FAIM1 gene, it is necessary to isolate this gene from pig for it is associated with energy metabolism, health and other

Corresponding Author: Yangzhi Zeng, Key Laboratory of Banna Mini-Pig Inbred Line of Yunnan Province, Yunnan Agricultural University, 650201 Kunming, Yunnan, China 
important biological functions of animals. But until today the porcine FAIM1 has not been reported yet. In 1980, the Banna Mini-pig Inbred line (BMI) was exploited by Yunnan Agricultural University based on the small ear pigs at Xishuangbanna, Yunnan Province, China. A pair of progenitors was a sow and her son with some degree inbreeding background. Then, the propagation was conducted by means of highly full sibling or parent offspring inbreeding and each generation underwent the strict selection. As heterozygotic genes were separated and recombined in the process of inbreeding, BMI has already owned six families and eighteen substrains with different phenotypes and genotypes. Due to their consistent genetic background and minor interindividual differences, BMI is considered as an ideal model organism for biological studies (Yu et al., 2004; Zeng and Zeng, 2005).

The objective of this study was to isolate the full length coding sequence of BMI FAIMI gene according to the conserved sequence information of cattle or other mammals and highly homologous swine ESTs sequence information, conduct sequence analysis and some necessary function analysis of established nucleotide sequence, finally examine the expression in a range of BMI tissues. These will provide a primary foundation for further research on this porcine gene.

\section{MATERIALS AND METHODS}

Samples collection, RNA extraction and first-strand cDNA synthesis: Three matured female BMI were slaughtered for sampling. Fresh tissues (Lymph node, midbrain, ovary, diencephalon, cerebrum, liver, kidney, spleen, heart, lung, nerve fiber, stomach, small intestine, large intestine, pancreas, skin, muscle and fat) were instantly frozen in liquid nitrogen and stored at $-80^{\circ} \mathrm{C}$ before use. Total RNA was extracted using the RNAiso Plus (TaKaRa, Dalian) according to the manufacturer's instructions. To remove genomic DNA contamination, total RNA was digested with RNase-free DNase I (TaKaRa, Dalian). Three micrograms of RNA were reverse transcribed with oligo $(\mathrm{dT})_{18}$ primer and $\mathrm{M}-\mathrm{MLV}$ reverse transcriptase (Invitrogen, USA).

Isolation of the BMI FAIM1 gene: The GenBank FAIM1 sequences for cattle (Accession No.: NM_001079626) and their highly homologous pig ESTs sequences: EW531886, EW483279, CN15846, FS676538, CN160386, EW333498, CN160763, EV898246 and EW530781 were used to design a primer pair to amplify the complete coding sequence of FAIM1 by using Primer Premier 5.0 Software. The primers for BMI FAMM1 gene were: 5'-GCC CTC GGA GCA CAG
ACT ATT T-3' and 5'-TCC TGA CCC TTG GAA CACT $\mathrm{AC} \mathrm{A-3}$ '. RT-PCR was performed to isolate the BMI FAIM1 using the pooled cDNAs from different tissues above. The $25 \mu \mathrm{L}$ reaction system was: $2.0 \mu \mathrm{L}$ cDNA (25 ng $\mu \mathrm{L}^{-1}$ ), $2.0 \mu \mathrm{L} 2.5 \mathrm{mM}$ mixed dNTPs (TaKaRa, Dalian), $2.5 \mu \mathrm{L} 10 \times$ Taq DNA polymerase buffer $\left(\mathrm{Mg}^{2+}\right.$ Plus), $0.5 \mu \mathrm{L} 10 \mu \mathrm{M}$ forward primer, $0.5 \mu \mathrm{L} 10 \mu \mathrm{M}$ reverse primer, $0.25 \mu \mathrm{L}$ Taq DNA polymerase $\left(5 \mathrm{U} \mu \mathrm{L}^{-1}\right.$, TaKaRa, Dalian) and $17.25 \mu \mathrm{L}$ sterile water. The PCR program initially started with $94^{\circ} \mathrm{C}$ denaturation for $2 \mathrm{~min}$ followed by 35 cycles of $94^{\circ} \mathrm{C} / 30 \mathrm{sec} 55^{\circ} \mathrm{C} / 40 \mathrm{sec} 72^{\circ} \mathrm{C} / 1.5 \mathrm{~min}$ then $72^{\circ} \mathrm{C}$ extension for $10 \mathrm{~min}$, finally $4^{\circ} \mathrm{C}$ to terminate the reaction. After the $\mathrm{PCR}$ the gene product was cloned into pMD18-T vector (TaKaRa, Dalian) and sequenced bidirectionally with the commercial fluorometric method. At least five independent clones were sequenced.

Bioinformatics analysis: Sequence analysis of BMI FAIM1 gene was performed using software in NCBI (http://www.ncbi.nlm.nih.gov) and ExPaSy (http://www. expsy.org). The cDNA sequence were predicted using the GenScan Software (http://genes.mit.edu/GENSCAN.html). The Blastp program and Conserved Domain Architecture Retrieval Tool were used to search for similar proteins and conserved domain respectively (http://www.ncbi.nlm. nih.gov/Blast). The alignment of the nucleotide sequences and deduced amino acid sequences were computed using ClusterX and the phylogenetic trees were computed using the ClustalX and Mega 4.0 Softwares with standard parameters. The theoretical isoelectric point (pI) and Molecular weight $(\mathrm{Mw})$ were predicted using the Compute $\mathrm{pI} / \mathrm{Mw}$ Tool (http://us.expasy.org/tools/pi_ tool.html). The putative signal peptide was predicted using the Signal P 4.0 server (http://www.cbs.dtu.dk/ services/SignalP/). The putative protein subcellular localization was predicted using PSort II (http://psort. hgc.jp/). Transmembrane topology prediction was performed using TMHMM-2.0 server (http://www.cbs. dtu.dk/services/TMHMM-2.0/). Secondary structures of deduced amino acid sequences were predicted with SOPMA (http://npsa-pbil.ibcp.fr/). Web-based microRNA (miRNA) predicting programs were used to locate conserved potential miRNA targets (http://www.mirbase. org/).

Semi-quantitative RT-PCR: To characterize the FAIM1 gene further, RT-PCR was conducted to determine its expression in $18 \mathrm{BMI}$ tissues. To eliminate the effect of cDNA concentration, we repeated the RT-PCR five times using 1-5 $\mu \mathrm{L}$ cDNA as templates. Researchers selected the housekeeping gene $18 S r R N A\left(\mathrm{NR}_{-} 002170\right)$ as a 
positive control. The control primers used were: 5'GGACATCTAAGGGCATCACAG-3' and 5'-AATTCC GATAACGAACGAGACT-3'. The BMI FAIM1 primers which were used to perform the semi-quantitative RT-PCR for tissue expression profile analysis were the same as the primers for isolation RT-PCR above. The PCR reactions were optimized for a number of cycles to ensure product intensity within the linear phase of amplification.

\section{RESULTS}

Isolation and identification of BMI FAIM1 cDNA: An FAIM1 fragment 791 bp of including the entire $603 \mathrm{bp}$ coding region plus $30 \mathrm{bp}$ of 5'-UTR and $158 \mathrm{bp}$ of 3 '-UTR was amplified (Fig. 1).

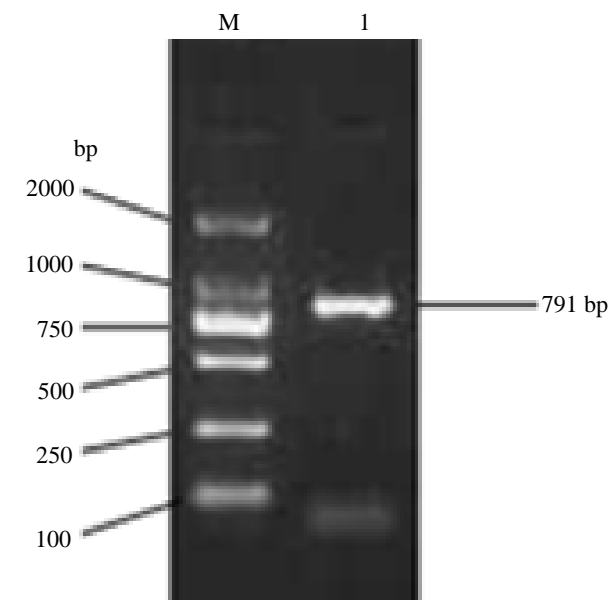

Fig. 1: RT-PCR result for BMI FAIM1 gene; M: DL2000 DNA marker; 1: PCR product of BMI FAIMlgene
This cDNA nucleotide sequence analysis using the BLAST software at NCBI server revealed that BMI FAIM1 gene was not homologous to any of the known porcine genes and it was then deposited into the GenBank database (Accession No.: JF271685). The sequence prediction was carried out using the GenScan Software and results showed that the $603 \mathrm{bp}$ cDNA sequence represent a single gene which encoded 200 amino acids. The complete CDS and the encoded amino acids were shown in Fig. 2.

Functional prediction of BMI FAIM1: The theoretical Isoelectric point $(\mathrm{pI})$ and Molecular weight $(\mathrm{Mw})$ of FAIM1 were computed using the compute $\mathrm{pI} / \mathrm{Mw}$ tool. The pI and the molecular weight of BMI FAIM1 are 4.90 and 22472.32, respectively. The result from Signal P revealed that there is has no putative signal peptide in BMI FAIM1. The potential protein subcellular localization prediction by Reinhardt's Method showed that BMI FAIM1 was probably located in the cytoplasm with up to $60.9 \%$ probability. Using a hidden Markov Model algorithm, transmembrane topology prediction made by TMHMM program (Moller et al., 2001) showed that BMI FAIM1 was not a potential transmembrane protein. The putative protein was also analyzed using prosite (http://expasy.org/prosite/). Four kinds sites were found which were Casein kinase II phosphorylation sites (3SgdD-6, 151-TagE-154), N-myristoylation sites (46GTtsGK-51,79-GAakTK-84, 167-GNhdCY-172, 184-GTihTL189), Protein kinase C phosphorylation sites (49-SgK-51, 107-S1K-109, 178-SgK-180) and Amidation sites (49-sGKR52, 145-nGKK-148, 178-sGKR-181) (Fig. 3).

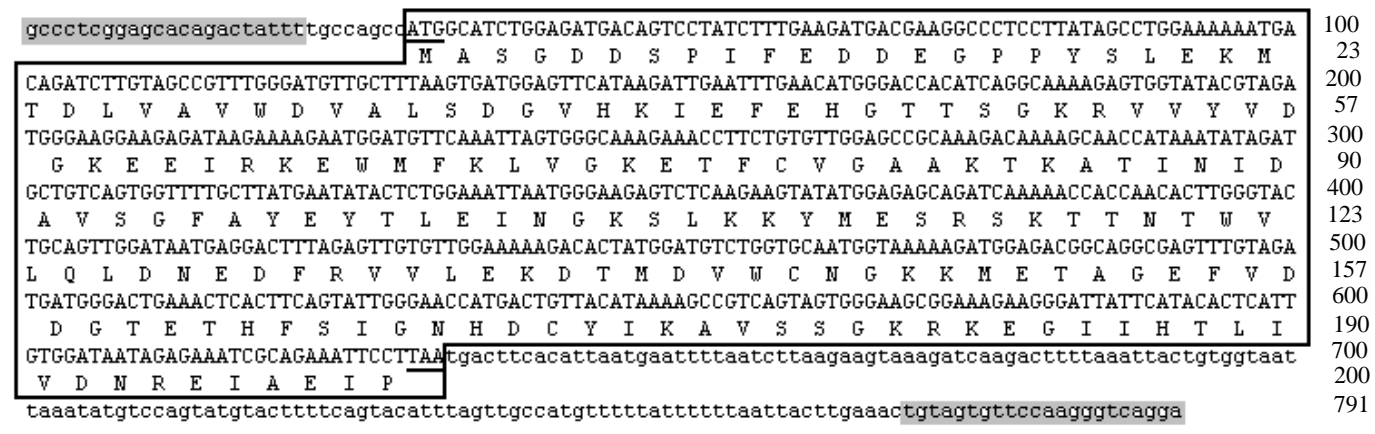

Fig. 2: The complete cDNA sequence and amino acid sequence of the protein encoded by FAIM1 (GenBank Accession No.: JF271685). ATG: start codon; TAA: stop codon; large pane: complete CDS and amino acid sequence; gray highlighted nucleotide sequence: primers

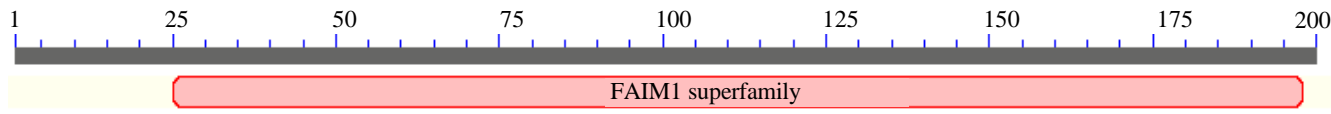

Fig. 3: The putative domains of the protein encoded by BMI FAIM1 

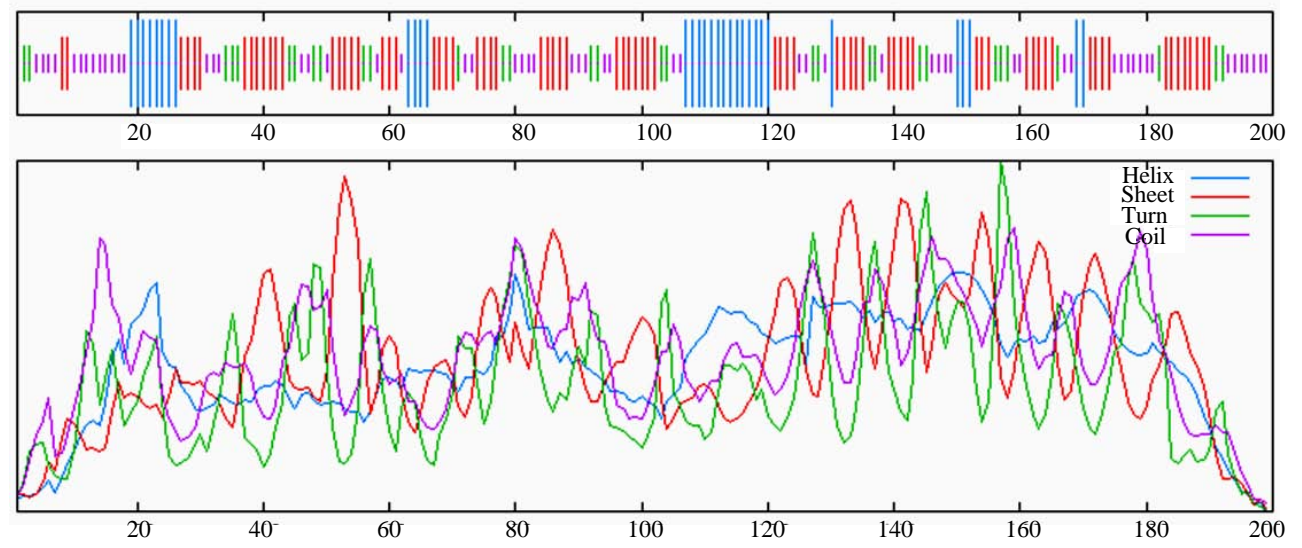

Fig. 4: The secondary structure of the BMI FAIM1 protein predicted by SOPMA

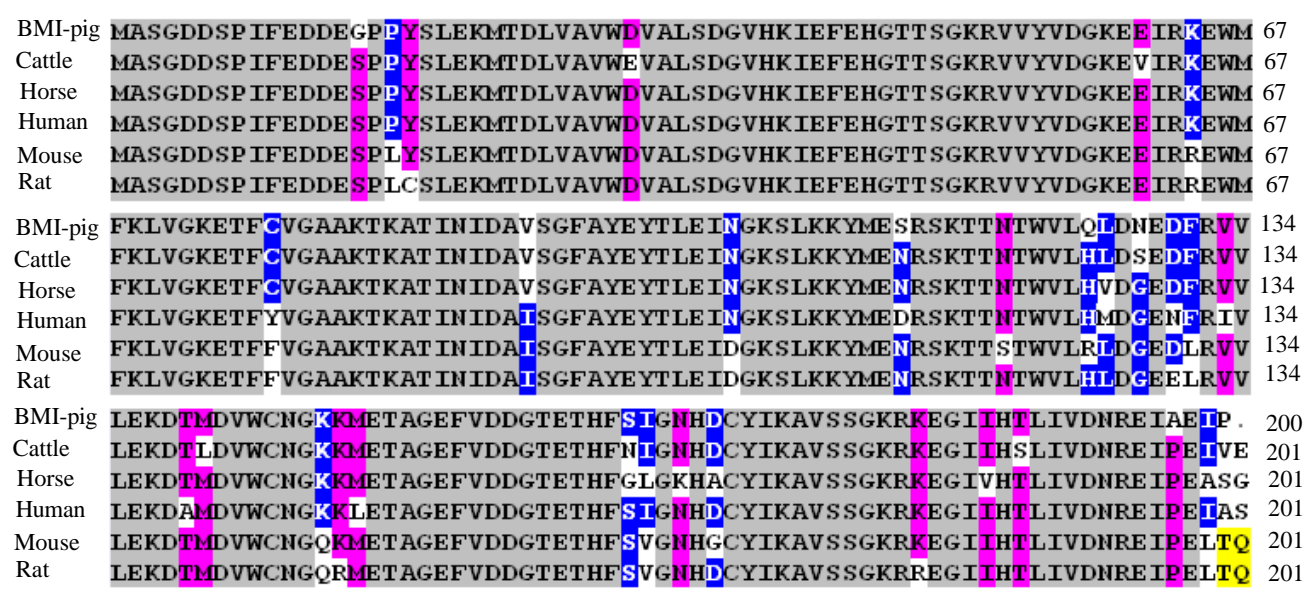

Fig. 5: Alignment of the protein encoded by the BMI-pig FAIM1 and five other types of FAIM1 from cattle (NP_001073094), horse (XP_001495579), human(NP_001028203), mouse (NP_001116323) and rat (XP_002730005)

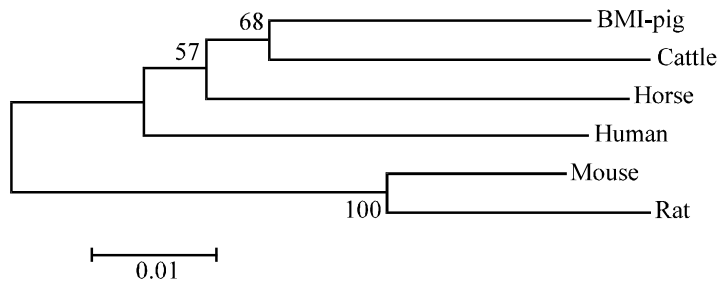

Fig. 6: Phylogenetic tree for FAIM1 proteins from 6 species

The prediction of secondary structure by SOPMA indicates that the deduced BMI FAIM1 consists of alpha helices (32AA), extended strands (77AA), beta turn (31AA) and random coils (60AA) (Fig. 4). MicroRNAs are noncoding single-stranded RNA molecules of 17-24 nucleotides that can regulate gene expression by binding to the coding region of target mRNAs (Bartel, 2004; Zeng et al., 2003). Researchers use web-based microRNA (miRNA) predicting programs to locate conserved potential miRNA targets: miRBase (http://www. mirbase.org/). The results showed three Sus scrofa microRNAs (ssc-miR-202, ssc-miR-130, ssc-miR-202) were found to have the predicted target sites (373-aagaaguaua uggagagca-355, 459-gugcaaugguaaaaag-474 and 315-ugcu uaugaauauacu-330) in the BMI FAIM1 sequence, respectively.

Further BLAST analysis revealed that the BMI FAIM1 protein has high homology with the Fas Apoptosis Inhibitory Molecule 1 (FAIM1) proteins of five species-cattle (95\%), horse (95\%), human (94\%), mouse $(92 \%)$ and rat (91\%) (Fig. 5). To evaluate the evolutionary relationships of BMI FAIM1 with other species, then we constructed a phylogenetic tree using DNAstar, Cluster, Mega and DNAman Software on the basis of the FAIM1 amino acid sequences. The phylogenetic tree analysis revealed that the BMI FAIM1 has a closer genetic relationship with the bovine FAIM1 than with those of horse, human, mouse and rat (Fig. 6). 


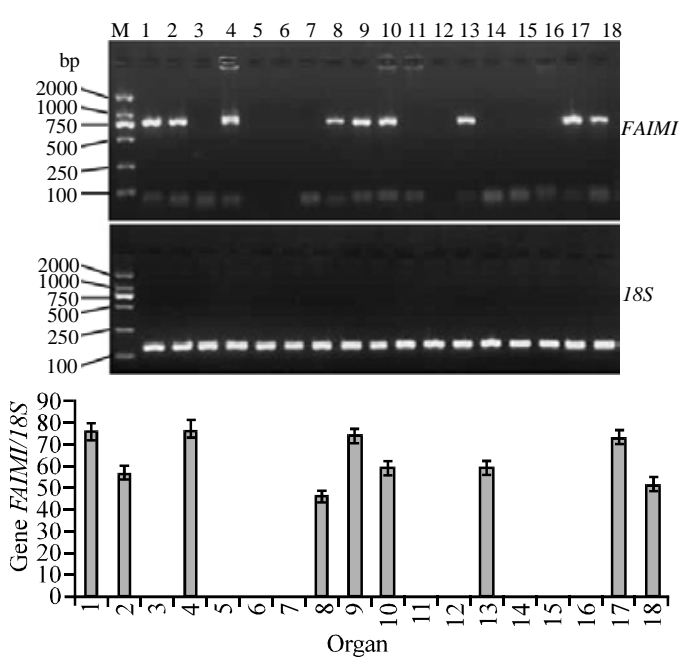

Fig. 7: Tissue expression distribution of BMI FAIM1 gene. The $18 \mathrm{~S}$ expression is the internal control. 1: Lymph node; 2: Midbrain; 3: Ovary; 4: Diencephalon; 5: Cerebrum; 6: Liver; 7: Kidney; 8: Spleen; 9: Heart; 10: Lung; 11: Nerve fiber; 12: Stomach; 13: Small intestine; 14: Large intestine; 15: Pancreas; 16: Skin; 17: Muscle; 18: Fat

mRNA tissue-specific expression profile: To check the relative expression levels of FAIM1 mRNA in various porcine tissues, semi-quantitative RT-PCR was performed in $18 \mathrm{BMI}$ tissues mentioned above. The continuously expressed gene, 18S was used and served as an endogenous reference for determination of targeted mRNA profiles. Result revealed that BMI FAIM1 gene was over-expressed in lymph node, diencephalon, heart, muscle, moderately expressed in midbrain, spleen, lung, small intestine, fat and almost not expressed in other 9 tissues (Fig. 7).

\section{DISCUSSION}

Comparative genomics determines the relationship of genome structure and function of different species. Researchers have learned a great deal about the function of human genes by examining their counterparts in simpler model organisms such as the mouse and some results have revealed that virtually all (99\%) of the protein-coding genes in humans align with homologues in mice and $>80 \%$ are clear 1:1 orthologs (Hardison, 2003; Liu et al., 2008). This extensive conservation in protein-coding regions implied that the same protein-coding sequences may be expected in different mammals including pig. From the isolation of swine FAIM1 gene, researchers can find that swine FAIM1 is highly homologous with FAIM1 of bovine, horse, human and other mammals. This further validated that comparative genomics method is one useful tool to isolate the unknown genes, especially the conserved coding region of genes for pig. From the alignment analyses for swine FAIM1 protein we also find BMI FAIM1 protein was not completely identity to cattle or other mammals. This implied that BMI FAIM1 will have some differences in functions to those of cattle, horse, human and other mammals.

The phylogenetic tree analysis revealed that the BMI FAIM1 gene has a closer genetic relationship with the bovine, horse and human FAIM1. Therefore, researchers can use bovine, horse and human as model organisms to study the pig FAIM1 gene or use pig as a model organism to study the bovine, horse and human FAIM1 genes.

MicroRNAs are small noncoding RNA. They play a role in gene expression regulation by inhibiting translation of their target mRNAs (Bartel, 2004; Zeng et al., 2003). Their target predictions showed that three Sus scrofa microRNAs (ssc-miR-202, sscmiR-130 and ssc-miR-202) were found to have the corresponding target sites (373-aagaaguauauggagagca355 , 459-gugcaau gguaaaaag-474 and 315ugcuuaugaauauacu-330) in the BMI FAIM1 sequence. Further investigation is needed to confirm whether corresponding miRNA molecules can regulate the FAIM1 gene expression in swine.

In this study, researchers not only cloned the CDS sequences of the BMI FAIM1 gene but also conducted the sequence analysis and tissue expression profiles analysis. From the tissue expression profile analysis, it can be seen that the gene was obviously differentially expressed in various tissues. As the researchers did not study functions at protein levels, there might be many possible reasons for differential expression of this porcine gene. The suitable explanation for this under current conditions is that the biological activities associated with the functions of the gene was demanded to a different extent in different tissues at the same time.

\section{CONCLUSION}

In this study, researchers first isolated BMI FAIM1 gene and performed necessary functional analysis and tissue expression profile analysis. Furthermore, several miRNAs were found to have the corresponding target sites in the coding sequence of BMI FAIM1 by theoretical prediction. The cDNA clone, sequence information and function analysis of BMI FAIM1 gene will be extremely important in elucidating the essential physiological function of FAIM1 protein using BMI and other miniature swines as experimental animal models in the future. 


\section{ACKNOWLEDGEMENT}

This study was supported by the National Natural Science Foundation of China (Grant No. 31160439) and Natural Science Foundation of Yunnan Province, China (Grant No. 2010ZC241). Jinglong Huo and Pei Wang contributed equally to this research.

\section{REFERENCES}

Bartel, D.P., 2004. MicroRNAs: Genomics, biogenesis, mechanism and function. Cell, 116: 281-297.

Brown, D. and J. Terris, 1996. Swine in Physiological and Pathophysiological Research. In: Advances in Swine in Biomedical Research, Tumbleson, M.E. and L.B. Schook, (Eds.). Plenum Press, New York, pp: 5-6.

Carey, T.E., D.L.V. Dyke and M.J. Worsham, 1993. Nonrandom chromosome aberrations and clonal populations in head and neck cancer. Anticancer Res., 13: 2561-2567.

Choi, C.Y., K. Reimers, C. Allmeling, S. Kall and Y.H. Choi and P.M. Vogt, 2007. Inhibition of apoptosis by expression of antiapoptotic proteins in recombinant human keratinocytes. Cell Transplant, 16: 663-674.

Cigudosa, J.C., N.Z. Parsa, D.C. Louie, D.A. Filippa and S.C. Jhanwar et al., 1999. Cytogenetic analysis of 363 consecutively ascertained diffuse large B-cell lymphomas. Genes Chromosomes Cancer, 25: $123-133$.

Douglas, W.R., 1972. Of pigs and men and research: A review of applications and analogies of the pig, sus scrofa, in human medical research. Space Life Sci., 3: $226-234$.

Hamaguchi, H., K. Suzukawa, K. Nagata, K. Yamamoto and F. Yagasaki and K. Morishita, 1997. Establishment of a novel human myeloid leukaemia cell line (HNT-34) with $t(3 ; 3)(\mathrm{q} 21 ; \mathrm{q} 26), \mathrm{t}(9 ; 22)$ (q34;q11) and the expression of EVI1 gene, P210 and P190 BCR/ABL chimaeric transcripts from a patient with AML after MDS with 3q21q26 syndrome. Br. J. Haematol., 98: 399-407.

Hardison, R.C., 2003. Comparative genomics. PLoS Biol., 1: e58-e58.

Harris, I., 1997. Variables in animal based research: Part 1. Phenotypic variability in experimental animals. Anzccart News, 10: 1-8.

Huo, J., S. Xu, K. Guo, Q. Zeng and K.P. Lam, 2009. Genetic deletion of faim reveals its role in modulating c-FLIP expression during CD95-mediated apoptosis of lymphocytes and hepatocytes. Cell Death Differ., 16: $1062-1070$.
Huo, J., S. Xu, and K.P. Lam, 2010. Fas apoptosis inhibitory molecule regulates $\mathrm{T}$ Cell receptormediated apoptosis of thymocytes by modulating Akt activation and Nur77 expression. J. Bilo. Chem., 285: 11827-11835.

Juneja, S., R. Lukeis, L. Tan, I. Cooper and G. Szelag et al., 1990. Cytogenetic analysis of 147 cases of nonHodgkin's lymphoma: non-random chromosomal abnormalities and histological correlations. Br. J. Haematol., 76: 231-237.

Larsen, M.O. and B. Rolin, 2004. Use of the Gottingen minipig as a model of diabetes, with special focus on type 1 diabetes research. ILAR. J., 45: 303-313.

Liu, G. Y., S.Z. Gao, C.R. Ge and X. Zhang, 2008. Molecular characterization of the encoding regions and tissue expression analyses for three novel porcine genes-HNRPA1, YIPF5 and UB2D2. Mol. Biol. Rep., 35: $519-526$.

Lunney, J.K., 2007. Advances in swine biomecical model genomics. Int. J. Biol. Sci., 3: 179-184.

Moller, S, M.D.R. Croning and R. Apweiler, 2001. Evaluation of methods for the prediction of membrane spanning regions. Bioinformatics, 17: 646-653.

Pekarsky, Y., E. Zabarovsky, V. Kashuba, H. Drabkin and A.A. Sandberg et al., 1995. Cloning of breakpoints in $3 q 21$ associated with hematologic malignancy. Cancer Genet. Cytogenet, 80: 1-8.

Petersen, I., M. Bujard, S. Petersen, G. Wolf and A. Goeze et al., 1997. imbalances in adenocarcinoma and squamous cell carcinoma of the lung. Cancer Res., 57: 2331-2335.

Reeds, P.J. and J. Odle, 1996. Pigs as models for nutrient functional interaction. In: Advances in Swine in Biomedical Research. Tumbleson, M.E. and L.B. Schook (Eds.). Plenum Press, New York, pp: 709-711.

Schneider, T.J., G.M. Fischer, T.J. Donohoe, T.P. Colarusso and T.L. Rothstein, 1999. A novel gene coding for a Fas apoptosis inhibitory molecule (FAIM) isolated from inducibly Fas-resistant B lymphocytes. J. Exp. Med., 189: 949-956.

Segura, M.F., C. Sole, M. Pascual, R.S. Moubarak and M. J. Perez-Garcia et al., 2007. The long form of Fas apoptotic inhibitory molecule is expressed specifically in neurons and protects them against death receptor-triggered apoptosis. J. Neurosci., 27: $11228-11241$.

Sole, C., X. Dolcet, M.F. Segura, H. Gutierrez and M.T. Diaz-Meco et al., 2004. The death receptor antagonist FAIM promotes neurite outgrowth by a mechanism that depends on ERK and NF-kappa B signaling. J. Cell Biol., 167: 479-492. 
Swindle, M.M., A.C. Smith, 1998. Comparative anatomy and physiology of the pig. J. Lab. Anim. Sci., 25: $168-188$.

Whang-Peng, J., T. Knutsen, A. Gazdar, S.M. Steinberg and H. Oie et al., 1991. Nonrandom structural and numerical chromosome changes in non-smallcell lung cancer. Genes Chromosomes Cancer, 3: $168-188$.

Wright, S., 1921. Systems of mating. I. The biometric relations between parent and offspring. Genetics, 6: 111-123.
Yu, P., L. Zhang, S. Li, Y. Li and J. Cheng et al., 2004. Screening and analysis of porcine endogenous retrovirus in Chinese banna minipig inbred line. Transplant. Proc., 36: 2485-2487.

Zeng, R. and Y.Z. Zeng, 2005. Molecular cloning and characterization of SLA-DR genes in the 133-family of the banna mini-pig inbred line. Anim. Genet., 36: 267-269.

Zeng, Y., R. Yi and B.R. Cullen, 2003. MicroRNAs and small interfering RNAs can inhibit mRNA expression by similar mechanisms. Proc. Natl. Acad. Sci., 100: 9779-9784. 